\title{
Two-body Coulomb problems with sources
}

\author{
G. Gasaneo ${ }^{1}$ and L. U. Ancarani ${ }^{2}$ \\ ${ }^{1}$ Departamento de Física, Universidad Nacional del Sur and Consejo Nacional de Investigaciones Científicas y Técnicas, \\ 8000 Bahía Blanca, Buenos Aires, Argentina \\ ${ }^{2}$ Laboratoire de Physique Moléculaire et des Collisions, Université Paul Verlaine-Metz, F-57078 Metz, France
}

(Received 26 July 2010; published 12 October 2010)

\begin{abstract}
The two-body Coulomb Schrödinger equation with different types of nonhomogeneities are studied. The particular solution of these nonhomogeneous equations is expressed in closed form in terms of a two-variable hypergeometric function. A particular representation of the latter allows one to study efficiently the solution in the asymptotic limit of large values of the coordinate and hence the associated physics. Simple sources are first considered, and a complete analysis of scattering and bound states is performed. The solutions corresponding to more general (arbitrary) sources are then provided and written in terms of more general hypergeometric functions.
\end{abstract}

DOI: 10.1103/PhysRevA.82.042706

PACS number(s): $03.65 . \mathrm{Nk}, 34.10 .+\mathrm{x}$

\section{INTRODUCTION}

A large variety of physical and mathematical problems can be formulated in terms of nonhomogeneous differential equations. The importance of this topic is put in evidence by the fact that it is considered in most of the undergraduate and graduate mathematical books for physicists, engineers, and so on. In electromagnetic theory, for example, the study of fields in presence of sources is directly related to the theory of nonhomogeneous differential equations [1]. Another very important branch of application is found in atomic physics; more precisely, nonhomogeneous equations play a fundamental role in scattering theory (see, e.g., Refs. [2,3]). The dynamics of the collision between two particles is ruled by the Schrödinger equation. Every collisional process can be associated, within the time-independent approach, to an initial condition corresponding to the solution of a simplified Hamiltonian where some of the interactions of the full Hamiltonian are neglected. The wave function satisfying the full Schrödinger equation is then generally written as the sum of the simplified and the scattering solution. This separation leads straightforwardly to a nonhomogeneous equation where the source is the product of the neglected interactions and the asymptotic solution [2,3]. This is exactly the line followed by, e.g., the exterior-complex-scaling approach [4,5]. Another, though different, application is found when building the asymptotical cosinelike stationary function within the $J$-matrix method [6-8]. Both approaches, which are widely and successfully used in treating a large variety of scattering problems, are based on the solution of nonhomogeneous (or driven) Schrödinger equations. The aim of this article is to present a closed form solution for a driven Schrödinger equation which includes a Coulomb interaction: This case is of fundamental importance for the atomic physics community.

Because of mathematical difficulties, only in few cases a direct solution of nonhomogeneous differential equations is considered. The standard method to deal with nonhomogeneous Schrödinger equations is based on the use of the Green function. In scattering studies, the solution can be written as an integral of the product of a Green function, the interaction and the asymptotic solution [2,3]. One of the advantages related to this approach is that it provides directly the appropriate asymptotic behavior of the solutions.
However, as shown by the authors (see Ref. [9]), it can lead to erroneous conclusions when dealing with long-range Coulomb interactions and inappropriate initial states. This shows the relevance of counting with exact and closed form solution for driven Schrödinger equations as those presented below.

In this article, a nonhomogeneous Schrödinger equation which includes a Coulomb interaction is solved in closed form. The formulation of the problem is presented in Sec. II A. The solution, as well as the driven term, are expanded in terms of spherical harmonics leading to a set of nonhomogeneous radial equations. For the Coulomb interaction and for particular types of sources (decreasing exponential times a power of the coordinate), a closed form solution can be found and expressed in terms of a generalized hypergeometric function in two variables (Sec. II B). A representation of this function as a series of product of Kummer and Gauss functions permits a derivation of an expression for the asymptotic behavior of the solutions (Sec. II C). A physical study of the Coulomb wave equation with a simple source is provided in Sec. III A for both positive and negative energies. The source considered is general enough to the used as the basic brick for almost any source. This is discussed in Sec. IIIB, where the formal solution is provided in terms of a generalized hypergeometric function of three variables. A summary and concluding remarks are given in Sec. IV. Atomic units $(\hbar=e=1)$ are used throughout.

\section{THE NONHOMOGENEOUS TWO-BODY COULOMB PROBLEM}

\section{A. Formulation of the problem}

As mentioned in the introduction different types of nonhomogeneous two-body Coulomb problem are related to a number of physical applications. We consider thus the following general problem:

$$
[H-E] \Psi(\mathbf{r})=\Phi_{0}(\mathbf{r}),
$$

where the Hamiltonian $H=-\frac{1}{2 \mu} \nabla^{2}+\frac{z_{1} z_{2}}{r}$ includes the kinetic energy (reduced mass $\mu$ ) and a Coulomb potential for two charges $z_{1}$ and $z_{2}$ (a subcase of interest is given by the case when one of the charges is zero). The potential being 
spherically symmetric, the wave function $\Psi(\mathbf{r})$ may be written with a partial waves decomposition

$$
\Psi(\mathbf{r})=\sum_{l, m} \mathcal{R}_{l m}(r) Y_{l}^{m}(\theta, \varphi)
$$

where $Y_{l}^{m}(\theta, \varphi)$ represent spherical harmonics [10] ( $l$ and $m$ represent the eigenvalues of, respectively, the angular momentum operators $\mathbf{L}$ and $\mathbf{L}_{z}$ ). Similarly, we may expand the source $\Phi_{0}(\mathbf{r})$ as

$$
\Phi_{0}(\mathbf{r})=\sum_{l, m} c_{l m} \mathcal{F}_{l}(r) Y_{l}^{m}(\theta, \varphi),
$$

where the radial part $\mathcal{F}_{l}(r)$ does not depend on $m$. Using the orthonormalization property of the spherical harmonics [10], the radial parts are then related through:

$$
\begin{aligned}
& {\left[-\frac{1}{2 \mu}\left(\frac{d^{2}}{d r^{2}}+\frac{2}{r} \frac{d}{d r}-\frac{l(l+1)}{r^{2}}\right)+\frac{z_{1} z_{2}}{r}-E\right] \mathcal{R}_{l}(r)} \\
& \quad=\mathcal{F}_{l}(r)
\end{aligned}
$$

where $\mathcal{R}_{l m}(r)=c_{l m} \mathcal{R}_{l}(r)$.

So far, no assumption has been made over the functions $\mathcal{F}_{l}(r)$ of the radial source. In order to maintain our study as general as possible, we consider now the following rather general formulation

$$
\mathcal{F}_{l}(r)=\frac{1}{r} \mathfrak{f}_{l}(r)=\frac{1}{r}\left(e^{-\lambda r} r^{l} \sum_{s=0}^{\infty} a_{l, s} r^{s}\right),
$$

which implies that $r \mathcal{F}_{l}(r)$ is analytic. No assumption is made on $\lambda$; when $\Re(\lambda)>0$, the source vanishes at large distances. The transformation

$$
\mathcal{R}_{l}(r)=\frac{\mathfrak{h}_{l}(r)}{r}
$$

converts Eq. (4) into the following

$$
\begin{aligned}
& {\left[-\frac{1}{2 \mu}\left(\frac{d^{2}}{d r^{2}}-\frac{l(l+1)}{r^{2}}\right)+\frac{z_{1} z_{2}}{r}-E\right] \mathfrak{h}_{l}(r)} \\
& =\mathfrak{f}_{l}(r)=e^{-\lambda r} r^{l} \sum_{s=0}^{\infty} a_{l, s} r^{s} .
\end{aligned}
$$

A particular case of this equation have been discussed and solved in terms of Laguerre function expansions [7,8].

The solutions of the nonhomogeneous differential equation (7) are given by the linear combination

$$
\mathfrak{h}_{l}(r)=\sum_{s=0}^{\infty} a_{l, s} h_{l, s}(r)
$$

where $h_{l, s}(r)$ are solutions of the simpler equation

$$
\begin{aligned}
& {\left[-\frac{1}{2 \mu}\left(\frac{d^{2}}{d r^{2}}-\frac{l(l+1)}{r^{2}}\right)+\frac{z_{1} z_{2}}{r}-E\right] h_{l, \sigma}(r)} \\
& \quad=a_{l, \sigma} e^{-\lambda r} r^{l+\sigma} .
\end{aligned}
$$

Here, we have used $\sigma$ instead of $s$ to have the opportunity to consider real or complex values of the power of the nonhomogeneity. The general solution of (9), $h_{l, \sigma}^{G}(r)$, can be written as a sum of the three terms

$$
h_{l, \sigma}^{G}(r)=A_{l}^{\text {Reg }} v_{l}^{\text {Reg }}(r)+A_{l}^{\text {Irreg }} v_{l}^{\text {Irreg }}(r)+h_{l, \sigma}^{P}(r) .
$$

The first two, $v_{l}^{\text {Reg }}(r)$ and $v_{l}^{\text {Irreg }}(r)$, are the solutions of the corresponding homogeneous equation, which is the wellknown Coulomb differential equation. Setting the energy $E=k^{2} /(2 \mu)$ hereafter taken as positive, and defining the Sommerfeld parameter $\alpha=z_{1} z_{2} \mu / k$, the solutions are given by $[3,11]$ :

$$
\begin{aligned}
& v_{l}^{\text {Reg }}(r)=N_{C}(l) r^{l+1} e_{1}^{i k r} F_{1}\left(\begin{array}{c}
i \alpha+l+1 \\
2 l+2
\end{array} \mid ;-2 i k r\right) \\
& v_{l}^{\text {Irreg }}(r)=N_{C}(l) r^{l+1} e^{i k r} U\left(\begin{array}{c}
i \alpha+l+1 \\
2 l+2
\end{array} \mid ;-2 i k r\right),
\end{aligned}
$$

where ${ }_{1} F_{1}(a, b ; z)$ and $U(a, b ; z)$ represent the regular and irregular solutions of the Kummer equation [12,13]. Both solutions, regular $\left[v_{l}^{\text {Reg }}(r)\right]$ and irregular $\left[v_{l}^{\text {Irreg }}(r)\right]$ at the origin, are real functions. The normalization constant

$$
N_{C}(l)=\frac{(2 k)^{l+1}}{2} \frac{|\Gamma(i \alpha+l+1)|}{\Gamma(2 l+2)} e^{-\frac{\pi}{2} \alpha},
$$

is chosen in such a way to have the following large distance behaviors

$$
\begin{gathered}
v_{l}^{\text {Reg }}(r) \longrightarrow \sin \left[\Phi_{C}(l)+\sigma_{C}(l)\right], \\
v_{l}^{\text {Irreg }}(r) \longrightarrow \cos \left[\Phi_{C}(l)+\sigma_{C}(l)\right],
\end{gathered}
$$

where

$$
\begin{gathered}
\Phi_{C}(l)=k r-\alpha \ln (2 k r)-\frac{\pi}{2} l \\
\sigma_{C}(l)=\operatorname{Arg}[\Gamma(i \alpha+l+1)] .
\end{gathered}
$$

One of the aims of this article is to provide useful analytic expressions for the particular solution $h_{l, \sigma}^{P}(r)$ and its asymptotic behavior. The second aim is to use these results as basic bricks for general type of sources (Sec. III).

To set the problem in a more appropriate form, we need to make a series of simple transformations in Eq. (9). We first introduce the change of function

$$
h_{l, \sigma}^{P}(r)=\frac{(-2 \mu)}{(-2 i k)^{\sigma+1}} a_{l, \sigma} e^{i k r} r^{l+1} f_{l, \sigma}(r)
$$

which leads to

$$
\begin{aligned}
& {\left[r \frac{d^{2}}{d r^{2}}+(2 l+2+2 i k r) \frac{d}{d r}-2 \alpha k+2 i k(l+1)\right] f_{l, \sigma}(r)} \\
& \quad=(-2 i k)^{\sigma+1} e^{-(\lambda+i k) r} r^{\sigma} .
\end{aligned}
$$

Second, the change of variable $u=-2 i k r$, transforms the previous into a nonhomogeneous Kummer-type equation [13] of the form

$$
\left[u \frac{d^{2}}{d u^{2}}+(c-u) \frac{d}{d u}-a\right] f_{l, \sigma}(u)=e^{\rho u} u^{\sigma},
$$

where the following definitions have been used

$$
a=i \alpha+l+1, \quad c=2 l+2, \quad \rho=\frac{1}{2}\left(1+\frac{\lambda}{i k}\right) .
$$

Equation (18) is the starting point of the present study. The two solutions of the corresponding homogeneous equation are known. The particular solution has been considered in the book of Babister [14], but no complete study was presented. Other authors, like, for example, Buchholz [15], have considered a particular type of nonhomogeneous Kummer-like equations. 


\section{B. A particular solution $h_{l, \sigma}^{P}(r)$ of the nonhomogeneous differential equation (18)}

The nonhomogeneous differential Eq. (18) can be solved first by expanding the exponential factor $e^{\rho u}$ and making then use of the procedure explained in the book of Babister [14]. A closed form of the particular solution of Eq. (18) is given by Eq. (4.219) [14]:

$$
f_{l, \sigma}(u)=u^{\sigma+1} \sum_{m=0}^{\infty} \sum_{n=0}^{\infty} \frac{\Gamma(\sigma+1+a+m+n) \Gamma(\sigma+1+n) \Gamma(\sigma+c+n)}{\Gamma(\sigma+1+a+n) \Gamma(\sigma+2+m+n) \Gamma(\sigma+1+c+m+n)} \frac{u^{n+m} \rho^{n}}{n !}
$$

as long as $\sigma$ is not a negative integer. This double series can be related to the series representation of a two variables hypergeometric function, named $\Theta^{(1)}$,

$$
\begin{aligned}
& \Theta^{(1)}\left(\begin{array}{c}
\left.a_{1}, a_{2}\left|b_{1}, b_{2}\right| ; x_{1}, x_{2}\right) \\
c_{1}\left|d_{1}, d_{2}\right|
\end{array}\right) \\
& =\sum_{m_{1}=0}^{\infty} \sum_{m_{2}=0}^{\infty} \frac{\left(a_{1}\right)_{m_{1}}\left(a_{2}\right)_{m_{2}}\left(b_{1}\right)_{m_{1}}\left(b_{2}\right)_{m_{1}+m_{2}}}{\left(c_{1}\right)_{m_{1}}\left(d_{1}\right)_{m_{1}+m_{2}}\left(d_{2}\right)_{m_{1}+m_{2}}} \frac{x_{1}^{m_{1}} x_{2}^{m_{2}}}{m_{1} ! m_{2} !},
\end{aligned}
$$

which we have introduced and discussed in Ref. [16] in a different context $\left((a)_{n}=\Gamma(a+n) / \Gamma(a)\right.$ represents the Pochhammer symbol in terms of Gamma functions). By direct comparison, the parameters $a_{i}, b_{i}, c_{1}, d_{i}$ and the variables $x_{i}$ $(i=1,2) \mathrm{read}$

$$
\begin{gathered}
a_{1}=\sigma+1, \quad a_{2}=1, \\
b_{1}=\sigma+c=2 l+2+\sigma, \\
b_{2}=c_{1}=a+\sigma+1=i \alpha+l+2+\sigma, \\
d_{1}=\sigma+2, \quad d_{2}=\sigma+c+1=2 l+3+\sigma . \\
x_{1}=\rho u=-(i k+\lambda) r, \quad x_{2}=u=-2 i k r .
\end{gathered}
$$

The particular solution $h_{l, \sigma}^{P}(r)$ of Eq. (9) can thus be expressed in terms of $\Theta^{(1)}$ as follows:

$$
h_{l, \sigma}^{P}(r)=(-2 \mu) a_{l, \sigma} \frac{1}{(\sigma+1)(2 l+2+\sigma)} e^{i k r} r^{l+\sigma+2} \Theta^{(1)}\left(\begin{array}{c}
\sigma+1,1 \mid 2 l+2+\sigma, i \alpha+l+2+\sigma \\
i \alpha+l+2+\sigma \mid 2+\sigma, 2 l+3+\sigma
\end{array} \mid ;-(i k+\lambda) r,-2 i k r\right)
$$

Even for real values of $\sigma, \lambda$, and $a_{l, \sigma}$, i.e., a real source, the particular solution $h_{l, \sigma}^{P}(r)$ is not necessarily real.

The $\Theta^{(1)}$ function was introduced in Ref. [16] to express the solution of a nonhomogeneous equation different from (18) and had equal variables $x_{1}=x_{2}$. In this sense, the appearance-here-of a $\Theta^{(1)}$ function, with $x_{1} \neq x_{2}$, is surprising. In Ref. [16] we have established a number of properties of the function $\Theta^{(1)}$, which is a Kampé de Fériet function [17], and provided series and integral representations. However, to study the asymptotic behavior of $h_{l, \sigma}^{P}(r)$, we found it necessary to derive another-more adequate-series representation which is derived in Ref. [18]. Indeed, in the limit $r \rightarrow \infty$ it is useful to uncouple as much as possible the variables $\rho$ and $u$ which will be, respectively, bound and large. Using the results given in Ref. [18], the solution $h_{l, \sigma}^{P}(r)$ can be expressed as

$$
\begin{aligned}
h_{l, \sigma}^{P}(r)= & (-2 \mu) a_{l, \sigma} \frac{e^{i k r} r^{l+\sigma+2}}{(\sigma+1)(2 l+2+\sigma)} \\
& \times \sum_{m=0}^{\infty} \frac{(2 i k r)^{m}}{m !} \frac{(\sigma+1)_{m}(i \alpha+l+2+\sigma)_{m}}{(2+\sigma)_{m}(2 l+3+\sigma)_{m}} \\
& \times{ }_{1} F_{1}\left(\begin{array}{c}
i \alpha+l+2+\sigma+m \\
2 l+3+\sigma+m
\end{array} \mid ;-2 i k r\right) \\
& \left.\times{ }_{2} F_{1}\left(\begin{array}{c}
-m, 2 l+2+\sigma \\
i \alpha+l+2+\sigma
\end{array}\right) ; \frac{1}{2}\left(1+\frac{\lambda}{i k}\right)\right) .
\end{aligned}
$$

This representation has the particularity of being a series of product of Kummer and Gauss functions dependingseparately - on the coordinate $r$ (or $u$ ) and the parameter $\rho=$ $\frac{1}{2}\left(1+\frac{\lambda}{i k}\right)$; the coupled dependence on these quantities, which was nontrivial in (23), was resummed in a particular manner. Note that, because of the negative integer as first parameter, the Gauss function ${ }_{2} F_{1}$ reduces to a polynomial of order $m$.

The special case $\lambda=0$, for which the exponential term of the source reduces to 1 , may be of interest. Mathematically, this situation has been considered by Babister (Eq. (4.233) of Ref. [14]), and the particular solution can be expressed in terms of a Lommel function. Setting $\lambda=0$ in formula (24), however, does not provide any substantial simplification since the Gauss function for argument equal $1 / 2$, even in our polynomial case, does not reduce to a simple function. For $\lambda \rightarrow \infty$ the sources vanishes exponentially. By asymptotic analysis of expression (24) it can be shown that the particular solution disappears as $\lambda^{-\sigma-1}$.

\section{The asymptotic behavior of the particular solution}

We now investigate the asymptotic behavior $(r \rightarrow \infty)$ of $h_{l, \sigma}^{P}(r)$; in this limit, both $x_{1}$ and $x_{2}$ are large, while the ratio $x_{1} / x_{2}$ takes the finite constant value $\rho=\frac{1}{2}\left(1+\frac{\lambda}{i k}\right)$. After a careful investigation, we found that among all the available representations of the $\Theta^{(1)}$ function, the one given by (24) 
considerably facilitates the asymptotic study. Using the results given in Ref. [18] with our parameters $a_{2}=1, d_{2}=b_{1}+1$, and $b_{2}=c_{1}$, it reads

$$
\begin{aligned}
\Theta^{(1)} \longrightarrow & \left(-x_{2}\right)^{-c_{1}} \frac{\Gamma\left(a_{1}+1\right) \Gamma\left(b_{1}+1\right)}{\Gamma\left(a_{1}+1+b_{1}-c_{1}\right)} \\
& \times{ }_{2} F_{1}\left(\begin{array}{c}
a_{1}, b_{1} \\
a_{1}+1+b_{1}-c_{1}
\end{array} \mid ; 1-\frac{x_{1}}{x_{2}}\right) \\
& +\frac{\Gamma\left(a_{1}+1\right) \Gamma\left(b_{1}+1\right)}{\Gamma\left(c_{1}\right)} e^{x_{2}} x_{2}^{c_{1}-b_{1}-1-a_{1}} \\
& \times{ }_{2} F_{1}\left(\begin{array}{c}
b_{1}, a_{1} \\
c_{1} \mid ; \frac{x_{1}}{x_{2}}
\end{array}\right)-\frac{a_{1} b_{1}}{x_{2}^{2}} \\
& {\left[e^{x_{1}}\left(\frac{x_{1}}{x_{2}}\right)^{b_{1}-c_{1}}+\frac{\Gamma\left(b_{1}\right)}{\Gamma\left(c_{1}-b_{1}\right)}\left(-x_{1}\right)^{-b_{1}} x_{2}^{c_{1}-b_{1}}\right] }
\end{aligned}
$$

The asymptotic form of the particular solution $h_{l, \sigma}^{P}(r)$, given by (23), becomes

$$
\begin{aligned}
h_{l, \sigma}^{P}(r) \longrightarrow & -2 \mu a_{l, \sigma}\left(C_{1} r^{-i \alpha} e^{i k r}+C_{2} r^{i \alpha} e^{-i k r}\right. \\
& \left.+C_{3} r^{\sigma+l} e^{-\lambda r}+C_{4} \frac{e^{i k r}}{r^{2+2 l}} r^{i \alpha}\right),
\end{aligned}
$$

where

$$
\begin{aligned}
C_{1}= & (2 i k)^{-(i \alpha+l+\sigma+2)} \frac{\Gamma(2 l+2+\sigma) \Gamma(1+\sigma)}{\Gamma(-i \alpha+l+2+\sigma)} \\
& \times \frac{|\Gamma(i \alpha+l+2+\sigma)|^{2}|\Gamma(i \alpha-l-1-\sigma)|^{2}}{|\Gamma(i \alpha+l+1)|^{2}|\Gamma(i \alpha-l)|^{2}} \\
& \times{ }_{2} F_{1}\left(\begin{array}{c}
\sigma+1,2 l+2+\sigma \\
-i \alpha+l+2+\sigma
\end{array} \mid ; \frac{1}{2}\left(1-\frac{\lambda}{i k}\right)\right) \\
C_{2}= & (-2 i k)^{i \alpha-l-\sigma-2} \frac{\Gamma(2 l+2+\sigma) \Gamma(1+\sigma)}{\Gamma(i \alpha+l+2+\sigma)} \\
& \times{ }_{2} F_{1}\left(\begin{array}{c}
\sigma+1,2 l+2+\sigma \mid ; \\
i \alpha+l+2+\sigma
\end{array} \mid ;\left(1+\frac{\lambda}{i k}\right)\right) \\
C_{4}=- & \frac{\Gamma(2 l+2+\sigma)}{\Gamma(i \alpha-l)}(i k+\lambda)^{-(2 l+2+\sigma)}(-2 i k)^{i \alpha-l-2} .
\end{aligned}
$$

For large values of $r$, the term associated to $C_{4}$ goes to zero faster than those related to $C_{1}$ and $C_{2}$ because of the power $1 / r^{2+2 l}$. Moreover, when $\Re(\lambda)>0$, the exponential decrease eliminates the term associated to $C_{3}$. Thus, in this case, the asymptotic behavior of $h_{l, \sigma}^{P}(r)$ reduces to

$$
h_{l, \sigma}^{P}(r) \longrightarrow-2 \mu a_{l, \sigma}\left(C_{1} r^{-i \alpha} e^{i k r}+C_{2} r^{i \alpha} e^{-i k r}\right),
$$

showing a linear combination of incoming and outgoing waves.

\section{APPLICATIONS}

In this section we shall investigate several physical situations involving different types of sources $\Phi_{0}(\mathbf{r})$.

\section{A. Simple source}

We take $\sigma=s$ with $s=0,1, \ldots$, and shall consider, separately, the cases of positive (respectively, negative) energies $E$, which correspond to an expected oscillating (respectively, exponentially decreasing) asymptotic behavior. For integer values of $\sigma$, the squared moduli in $C_{1}$ reduce to 1 ; hence, if $\lambda$ is real, we have $C_{1}=C_{2}^{*}$. Below we shall consider that $\Re(\lambda)>0$, so the asymptotic form is given by (27). For convenience we set

$$
{ }_{2} F_{1}\left(\begin{array}{c}
s+1,2 l+2+s \\
i \alpha+l+2+s
\end{array} \mid ; \frac{1}{2}\left(1+\frac{\lambda}{i k}\right)\right)=\left.\right|_{2} F_{1} \mid e^{i \Delta} .
$$

\section{Positive energies}

Consider the simple case of the radial source given by (9), i.e., $a_{l, s} e^{-\lambda r} r^{l+s}$, where $\lambda$ is real and positive and $l$ general. After some manipulations, the asymptotic form (27) of the particular solution can be written as

$$
h_{l, s}^{P}(r) \longrightarrow N_{\text {source }} \cos \left[\Phi_{C}(l+s+2)+\delta(l, s, \lambda)\right] .
$$

with

$$
\begin{gathered}
\delta(l, s, \lambda)=\sigma_{C}(l+s+1)-\Delta(l, s, \lambda), \\
N_{\text {source }}=-2 \mu a_{l, \sigma} \frac{(1)_{s}(2 l+2)_{s}}{(2 l+2)_{2 s+2}} \frac{\left|{ }_{2} F_{1}\right|}{N_{C}(l+s+1)} .
\end{gathered}
$$

where the functions $N_{C}(l), \Phi_{C}(l)$, and $\sigma_{C}(l)$ are defined by (12), (14), and (15), respectively.

In (29), the phase $\Phi_{C}(l+s+2)$ appears shifted when compared to $\Phi_{C}(l)$ because of the presence of the power $r^{s}$ in the source. In addition, the first contribution to the phase shift $\delta(l, s, \lambda)$, is the Coulomb phase $\sigma_{C}(l+s+1)$ with a modified $l$, again due to the presence of $r^{s}$ in the source. The second contribution, $-\Delta(l, s, \lambda)$, depends not only on $s$ but also on the $\lambda$ parameter related to the exponential decay of the source.

While the prefactor $N_{\text {source }}$ depends on the source itself through $a_{l, \sigma}, \lambda$, and $s$, we could introduce some normalization in such a way that the solution $h_{l, s}(r)$ behaves at large values of the coordinates as a pure cosine function with unit amplitude. The regular $v_{l}^{\text {Reg }}(r)$ and irregular $v_{l}^{\text {Irreg }}(r)$ solution of the homogeneous equation are real functions. According to Eq. (29), for real values of $a_{l, \sigma}$, i.e., a real source, the particular solution $h_{l, \sigma}^{P}(r)$ is also real. This is a priori not at all obvious.

In Fig. 1 we plot $h_{l, s}^{P}(r)$ as a function $r$, for positive energy $E=1, z_{1} z_{2}=-1, \mu=1, a_{l, s}=1, \lambda=1, l=0$, and for $s=0,1$. The asymptotic limit of the solution, Eq. (29), was included in the plot for comparison. The first thing to be noted is that the solution $h_{l, s}^{P}(r)$ is indeed a real definite function. In addition, it is regular at the origin and has an oscillatory behavior similar to $v_{l}^{R e g}(r)$ aside from a phase shift. The fact that the function is real and its maxima are bounded illustrates the cosine asymptotic behavior (29). Since for $\lambda>0$ 


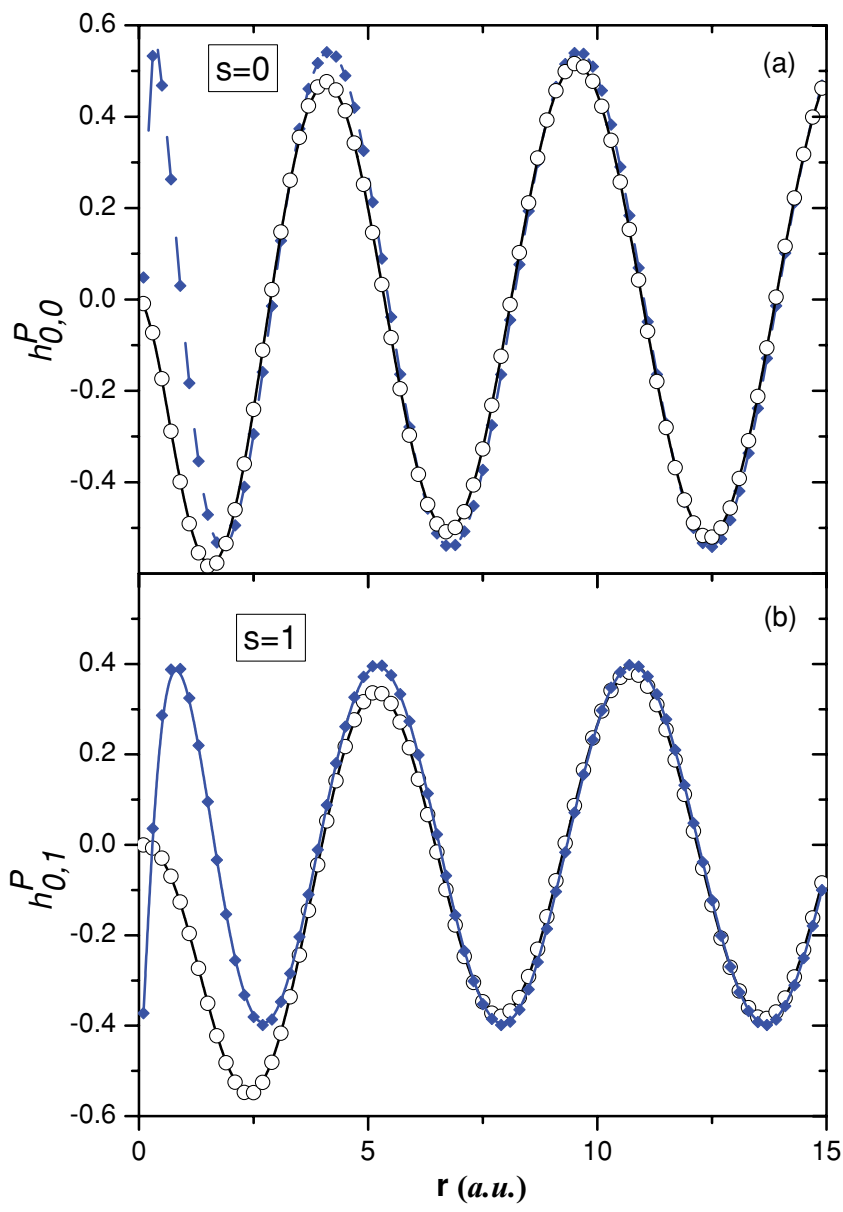

FIG. 1. (Color online) The particular solution $h_{l, s}^{P}(r)$ (solid lines and open circles) and its asymptotic form (solid lines and diamonds) are plotted as a function of $r$ for $l=0, z_{1} z_{2}=-1, \mu=1, a_{l, s}=1$ and for positive energy $E=1$; (a) $s=0$ and (b) $s=1$.

the source decreases exponentially for large $r$, we expect the solution to possess the Coulomb-like asymptotic behavior related to the homogeneous equation, i.e., the behaviors (13a) and (13b).

It is interesting to notice that even in the case $s=0$, a phase shift with respect to the corresponding Coulomb phase $\sigma_{C}(l)$ appears. We can understand the physics of the problem assuming that there are two contributions to the flux of particles described by the wave function: one is an external stationary flux, while the other is the one generated by the source. Indeed, asymptotically, the wave function can be written as a cosine function [see Eq. (29)] with the Coulombic phase $\Phi_{C}$ shifted by the quantity $\delta(l, s, \lambda)$ which takes into account the difference between the contributions to the whole flux. Playing with the parameters $\lambda, k$, and $s$, the phase shift $\delta(l, s, \lambda)$ can be adjusted to the one of the external flux, i.e., reduced to the Coulomb phase shift $\sigma_{C}(l)$. With such a choice, no contribution from the source would be observed at large distances, and $h_{l, s}^{P}(r)$ would behave exactly as the second solution of the homogeneous functions $v_{l}^{\text {Irreg }}(r)$ of (10). Thus, a kind of Ramsauer-Townsend effect [3] associated to the source would be observed.

\section{Negative energies}

For negative energies $E$, and in the case of an attractive potential $z_{1} z_{2}<0$, we expect the solution $h_{l, s}^{P}(r)$ to have an exponentially decreasing behavior at large values of the coordinate $r$. One way of getting this behavior is obtained through a rotation to the complex plane of the momentum; indeed, replacing $k$ by $i \kappa$ with $\kappa>0$, the exponential factor $e^{i k r}$ of (24) is transformed into $e^{-\kappa r}$. This analytic continuation also leads to $i \alpha=\frac{z_{1} z_{2} \mu}{\kappa}$ and $E=-\kappa^{2} / 2 \mu$.

Before considering the particular solution $h_{l, s}^{P}(r)$, let us have a look at the regular $v_{l}^{R e g}(r)$ and the irregular $v_{l}^{\text {Irreg }}(r)$ solutions. It can be easily verified that both functions diverge exponentially at large distances for general values of the parameter $\kappa$. The problem can be avoided, as done generally in quantum mechanics (e.g., hydrogen atom) [10], by choosing particular values of $\kappa$ :

$$
\kappa=-\frac{z_{1} z_{2} \mu}{n} ; \quad n=l+1+n_{r} \quad\left(n_{r}=0,1,2, \ldots\right),
$$

where $n$ is a quantum number. In this case, the ${ }_{1} F_{1}$ and the $U$ functions of (11a) and (11b) reduce to polynomials, and the regular and irregular solutions are well behaved at large distances.

The above choice of $\kappa$ does not guarantee that the particular solution $h_{l, s}^{P}(r)$ is also well behaved. On replacement of $k$ by $i \kappa$ in the asymptotic expression (26), we observe that $C_{2}$ must vanish in order to have a bound behavior for $h_{l, s}^{P}(r)$. This implies

$$
\begin{aligned}
& \frac{1}{\Gamma\left(\frac{z_{1} z_{2} \mu}{\kappa}+l+2+s\right)} \\
& \quad \times{ }_{2} F_{1}\left(\begin{array}{c}
s+1,2 l+2+s \\
\frac{z_{1} z_{2} \mu}{\kappa}+l+2+s
\end{array} \mid ; \frac{1}{2}\left(1-\frac{\lambda}{\kappa}\right)\right)=0 .
\end{aligned}
$$

Consider first $\kappa=\kappa_{N}$ values such that $\frac{z_{1} z_{2} \mu}{\kappa_{N}}+l+2+s=$ $-N$ where $N$ is a non-negative integer, expression (32) becomes

$$
\left(1-\frac{\lambda}{\kappa_{N}}\right)^{N+1}{ }_{2} \tilde{F}_{1}\left(\begin{array}{c}
s+1+N+1,2 l+2+s+N+1 \\
N+2
\end{array} \mid ; \frac{1}{2}\left(1-\frac{\lambda}{\kappa_{N}}\right)\right)=0
$$


where ${ }_{2} \tilde{F}_{1}$ is the regularized ${ }_{2} F_{1}$ function. Generally, condition (33) will be satisfied only for very particular values of $\lambda$ and are therefore of limited interest. For the obvious case $\kappa_{N}=\lambda$, only one particular solution exists and is given by

$$
\begin{aligned}
h_{l, s}^{P}(r)= & (-2 \mu) a_{l, s} \frac{e^{-\lambda r} r^{l+s+2}}{(s+1)(2 l+2+s)} \\
& \times{ }_{2} F_{2}\left(\begin{array}{c}
-N, 1 \\
\sigma+2,2 l+3+\sigma
\end{array} \mid ; 2 \lambda r\right),
\end{aligned}
$$

i.e., a decreasing exponential times a polynomial of order $l+$ $s+2+N ; N=0$ will give the lowest energy level. Note that, in this case, the $\kappa_{N}$ values may also correspond to well-behaved solutions of the homogeneous equation [see Eq. (31)].

For general values of $\lambda$, the condition (32) is satisfied for discrete $\kappa=\kappa_{M}$ values satisfying

$$
{ }_{2} F_{1}\left(\begin{array}{c}
s+1,2 l+2+s \\
\frac{z_{1} z_{2} \mu}{\kappa_{M}}+l+2+s
\end{array} \mid ; \frac{1}{2}\left(1-\frac{\lambda}{\kappa_{M}}\right)\right)=0 .
$$

These $\kappa_{M}$ values will depend on $\lambda, s$, and $l$; they cannot be given in closed form since there are no analytic formulas for the roots of the Gauss function for general parameters (furthermore, $\kappa_{M}$ appears not only in the argument but also in the third parameter). A given $\kappa_{M}$ value will generally lead to exponentially divergent homogeneous solutions. Of course, it may happen that $\lambda$ coincides with one of the values $\kappa_{N}$ described above. In that case, one of the negative energy states will correspond to the solution (34) while all the others will be associated to the zeros coming from condition (35). In all cases, the particular solution $h_{l, s}^{P}(r)$ corresponds to a bound state satisfying Eq. (9), thus validating the analytic continuation $k \rightarrow i \kappa$.

In Fig. 2, the function ${ }_{2} F_{1}$ of condition (35) is shown as a function of $\kappa$ for $\lambda=0.56, s=0, l=0, \mu=1$, and $z_{1} z_{2}=$ -1 . We observe the occurrence of nine zeros. However, there are an infinite number of them due to the fact that there are infinite branches of the tan-like function in the region 0 to 1 of $\kappa$; they accumulate close to $\kappa=0$.

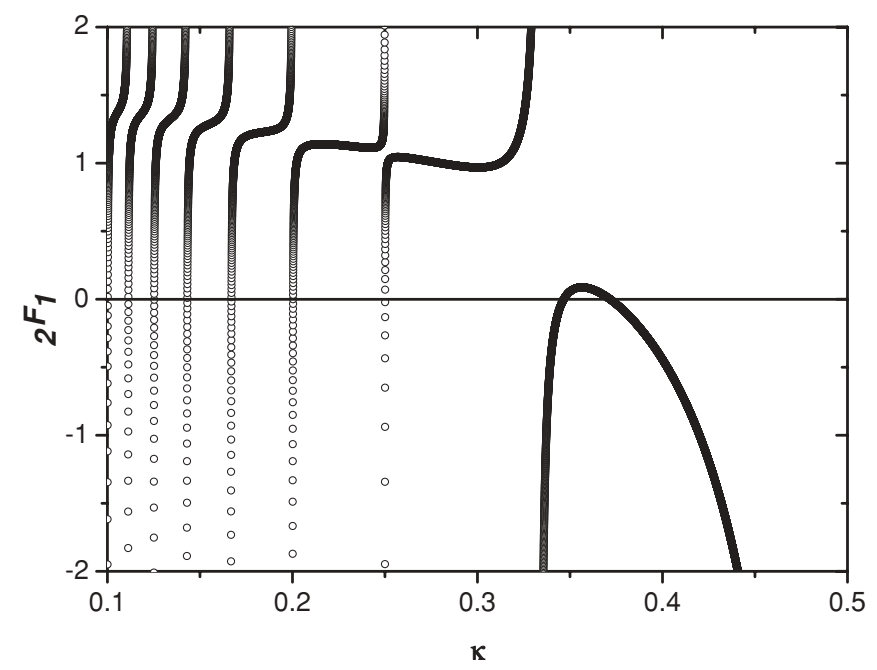

FIG. 2. The function of Eq. (35) (which defines the bound states of the particular solution of the nonhomogeneous equation) is plotted against $\kappa$ (open circles). The plot corresponds to $l=0, s=0, z_{1} z_{2}=$ -1 , and $\mu=1$.
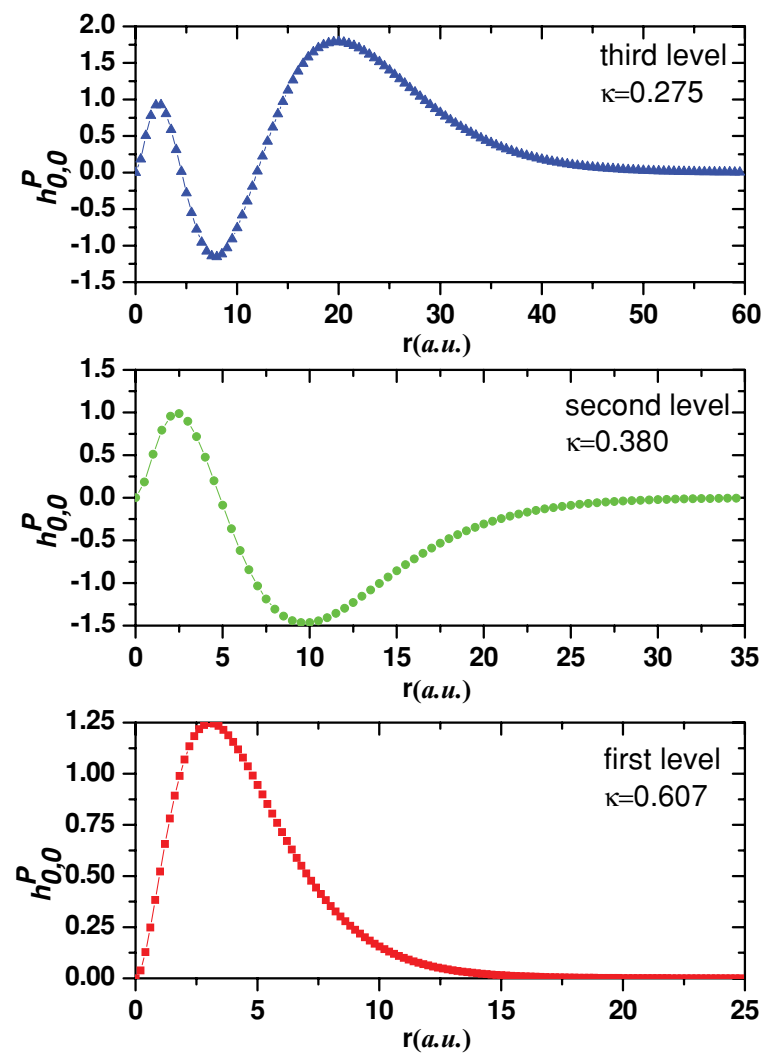

FIG. 3. (Color online) The function $h_{l, s}^{P}(r)$ is plotted as a function of $r$ for $l=0, s=0, z_{1} z_{2}=-1, \mu=1$, and $a_{l, s}=1$. The first three bound states corresponding to $\kappa=0.6073,0.3802$, and 0.2758 are presented.

In Fig. 3, we plot the first three bound states of $h_{l, s}^{P}(r)$ corresponding to $\kappa=0.607,0.380$, and 0.275 , and with $\lambda=$ $0.56, s=0, l=0, \mu=1, a_{l, s}=1$, and $z_{1} z_{2}=-1$. As we can see the number of nodes increases by 1 as happens with the hydrogen atom.

\section{B. General source}

We will now show how to treat the problem of a general source $\mathfrak{f}_{l}(r)$ in the nonhomogeneous equation (7). The case of a simple source studied in the previous section will be used to build the solution for the general source. Take again the case of positive energies $E$.

Consider first the following definition for the function $\mathfrak{f}_{l}(r)$

$$
\begin{aligned}
\mathfrak{f}_{l, p}(r)= & \frac{2 \gamma}{\Gamma(2 l+2)}\left[\frac{\Gamma(1-p)}{\Gamma(2 l+2-p)}\right]^{-\frac{1}{2}} \\
& \times \sum_{s=0}^{\infty} \frac{(p)_{s}}{s !(2 l+2)_{s}}\left(e^{-\lambda r}(2 \gamma r)^{s+l}\right), \\
= & \frac{1}{\Gamma(2 l+2)}\left[\frac{\Gamma(1-p)}{\Gamma(2 l+2-p)}\right]^{-\frac{1}{2}} \frac{e^{-\lambda r}}{r}(2 \gamma r)^{l+1} \\
& \times{ }_{1} F_{1}\left(\begin{array}{c}
p \\
2 l+2
\end{array} \mid ; 2 \gamma r\right),
\end{aligned}
$$

the coefficients of (5) being chosen in order to construct a Kummer function. This particular choice is dictated by the 
following considerations. We take $\gamma=\lambda$ and choose $p$ as a negative integer $-n$, so that the Kummer function reduces to a polynomial [13]; in this case, the source $\mathfrak{f}_{l}(r)$ reads

$$
\begin{aligned}
\mathfrak{f}_{l, n}(r) & =\frac{1}{r}\left[\sqrt{\frac{n !}{\Gamma(2 l+2+n)}} e^{-\lambda r}(2 \lambda r)^{l+1} L_{n}^{(2 l+1)}(2 \lambda r)\right] \\
& =\frac{1}{r} \varphi_{n, l}(\lambda, r),
\end{aligned}
$$

where $L_{n}^{(\alpha)}(z)$ are Laguerre polynomials [13]. It is known that the functions $\varphi_{n, l}(\lambda, r)$ form an orthogonal and complete set of functions and satisfy the following relations [13]:

$$
\begin{aligned}
& \int_{0}^{\infty} \varphi_{n, l}(\lambda, r) \frac{1}{r} \varphi_{n^{\prime}, l}(\lambda, r) d r=\delta_{n, n^{\prime}}, \\
& \sum_{n} \varphi_{n, l}\left(\lambda, r^{\prime}\right) \frac{1}{r} \varphi_{n, l}(\lambda, r)=\delta\left(r-r^{\prime}\right) .
\end{aligned}
$$

Because of these properties, a general function radial $\mathcal{F}_{l}(r)$ of the source (3), may be expressed through an expansion

$$
\mathcal{F}_{l}(r)=\frac{1}{r} \mathfrak{f}_{l}(r)=\sum_{n} d_{n, l} \varphi_{n, l}(\lambda, r),
$$

and hence the general source given by (3) as

$$
\Phi_{0}(\mathbf{r})=\frac{1}{r} \sum_{l, m} c_{l, m} d_{n, l} \varphi_{n, l}(\lambda, r) Y_{l}^{m}(\theta, \varphi) .
$$

Now, from our study in Sec. II, we know the solution of the nonhomogeneous differential equation (7) with its source $\mathfrak{f}_{l, p}(r)$ defined by (36); according to (8) the particular solution is given by

$$
\begin{aligned}
\mathfrak{h}_{l, s, p}^{P}(r)= & \frac{1}{\Gamma(2 l+2)}\left[\frac{\Gamma(1-p)}{\Gamma(2 l+2-p)}\right]^{-\frac{1}{2}} \\
& \times(2 \gamma)^{l+1} \sum_{s=0}^{\infty} \frac{(p)_{s}}{s !(2 l+2)_{s}}(2 \gamma)^{s} h_{l, s}^{P}(r)
\end{aligned}
$$

and replacing the definition $(23)$ of $h_{l, s}^{P}(r)$ in terms of $\Theta^{(1)}$, we obtain

$$
\begin{aligned}
\mathfrak{h}_{l, s, p}^{P}(r)= & {\left[\frac{\Gamma(1-p)}{\Gamma(2 l+2-p)}\right]^{-\frac{1}{2}} \frac{(-2 \mu) a_{l, s}}{\Gamma(2 l+2)} r e^{i k r}(2 \gamma r)^{l+1} \sum_{s=0}^{\infty} \frac{(p)_{s}(1)_{s}}{s !(2)_{s}(2 l+3)_{s}}(2 \gamma r)^{s} } \\
& \times \Theta^{(1)}\left(\begin{array}{c}
s+1,1 \mid 2 l+2+s, i \alpha+l+2+s \\
i \alpha+l+2+s \mid 2+s, 2 l+3+s
\end{array} \mid ;-(i k+\lambda) r,-2 i k r\right) .
\end{aligned}
$$

Using the power series definition of $\Theta^{(1)}$ [16] and some properties of Pochhammer symbols, this solution may be also represented in terms of a three-variable hypergeometric function

$$
\mathfrak{h}_{l, s, p}^{P}(r)=\left[\frac{\Gamma(1-p)}{\Gamma(2 l+2-p)}\right]^{-\frac{1}{2}} \frac{(-2 \mu) a_{l, s}}{\Gamma(2 l+2)} e^{i k r} r(2 \gamma r)^{l+1} \Xi\left(\begin{array}{c}
1, p|1,2 l+2| i \alpha+l+2 \\
2 l+2|i \alpha+l+2| 2,2 l+3
\end{array} \mid ;-(i k+\lambda) r,-2 i k r, 2 \gamma r\right),
$$

where $\Xi$ is the following generalized hypergeometric function defined as

$$
\Xi\left(\begin{array}{c}
a_{1}, a_{2}\left|b_{1}, b_{2}\right| c_{1} \\
d_{1}\left|e_{1}\right| f_{1}, f_{2}
\end{array} \mid ; x_{1}, x_{2}, x_{3}\right)=\sum_{m_{1}=0}^{\infty} \sum_{m_{2}=0}^{\infty} \sum_{m_{3}=0}^{\infty} \frac{\left(a_{1}\right)_{m_{2}}\left(a_{2}\right)_{m_{3}}\left(b_{1}\right)_{m_{1}+m_{3}}\left(b_{2}\right)_{m_{1}+m_{3}}\left(c_{1}\right)_{m_{1}+m_{2}+m_{3}}}{\left(d_{1}\right)_{m_{3}}\left(e_{1}\right)_{m_{1}+m_{3}}\left(f_{1}\right)_{m_{1}+m_{2}+m_{3}}\left(f_{2}\right)_{m_{1}+m_{2}+m_{3}}} \frac{x_{1}^{m_{1}}}{m_{1} !} \frac{x_{2}^{m_{2}}}{m_{2} !} \frac{x_{3}^{m_{3}}}{m_{3} !},
$$

which is convergent for any value of the variables $x_{i} \in[0, \infty)$.

In summary, according with the previous expressions, the particular solution of the general nonhomogeneous Eq. (1) is given by

$$
\Psi^{P}(\mathbf{r})=\sum_{l, m} c_{l, m} d_{n, l} \frac{\mathfrak{h}_{l, s, n}^{P}(r)}{r} Y_{l}^{m}(\theta, \varphi),
$$

where the particular solution $\mathfrak{h}_{l, s, n}^{P}(r)$, corresponding to the source $\mathfrak{f}_{l, n}(r)$ of Eq. (37), is given by the compact expressions (42) or (43) with $p=-n$. This is one of the main results of this article. It provides the particular solution corresponding to the emission of particles from any source in presence of a Coulomb potential. The solutions of the corresponding homogeneous equation can be summed to represent the general solutions of the nonhomogeneous differential equation. The function $\Psi(\mathbf{r})$ contains the dynamics of the problem.

\section{SUMMARY AND CONCLUDING REMARKS}

In this article we provide explicit solutions for the nonhomogeneous Schrödinger equation containing a Coulomb interaction, with different types of nonhomogeneities. The simplest case, corresponding to a driven term defined by the product of an exponential $e^{-\lambda r}$ and a power $r^{\sigma}$, is studied in details. The particular solution is expressed in closed form in terms of the two-variable hypergeometric function $\Theta^{(1)}$ [16]. The use of a series representation in terms of products of Kummer and Gauss functions separates, term by term, the coordinate $r$ from the parameter $\rho$ which is related to $\lambda$ and the energy appearing in the exponential part of the source; it is also particularly useful to derive the asymptotic limit of the wave function for large values of the coordinate $r$. A complete discussion about the dynamics of the system contained in the particular solution was presented for both positive and negative energies. For sources with real positive values of $\lambda$ and non-negative integer $s$ values of $\sigma$, the asymptotic 
behavior described by the scattering solution corresponds to a superposition of incoming and outgoing waves; these can be expressed as a standing cosine wave function with a nonzero phase shift between the incoming and outgoing parts. This phase shift is due not only to the one produced by the Coulomb potential but also to the phase shift observed between an external flux of particles and that generated by the source. The combination of the particular solution with the general solution of the corresponding homogeneous equation can be chosen in such a way to attribute to the total wave function different type of fluxes.

For negative energy, bound states can be constructed for any value of $\lambda$. Through an analytic continuation of the momentum, imaginary values of $k$ can be found in such a way to enforce the scattering solution to decrease exponentially at large distances. In that way particular values of $k$ are obtained leading to a discretization of the energy. For these energy values the homogeneous solutions generally diverges at large distances. There are other possible values of $k$ that allow the existence of one bound state both for the solutions of the homogenous and the nonhomogeneous equation, but that state appears only for very particular $\lambda$ values.

Very interestingly, we found that for real sources, the particular solution of the nonhomogeneous Coulomb Schrödinger equation is real, as are the well-known regular and irregular solutions of the homogeneous equation. This is true for both positive and negative energies. The results obtained for positive energies were used, subsequently, as basic bricks to give closed form expressions for the solution of Schrödinger equation with general sources. Given the expansion of the source in terms of spherical harmonics and Laguerre basis functions, the solution for a Schrödinger equation can be written as a sum of three-variable hypergeometric functions.
The study presented here can be applied to the study of wide variety of physical processes and into the understanding and extension of very important methods used for the description of scattering problems. One such example is the exterior-complex-scaling method which is widely used in the description of diverse scattering processes; the method is based on the solution of a driven Schrödinger equation. Indeed, within this approach, the wave function describing the scattering process is separated into two terms. One of them is an approximate state which satisfies a wave equation not including all the interactions present in the full Hamiltonian. The second term, which contains the information about the dynamics of the collision, satisfies a driven Schrödinger equation and is expected to provide an outgoing behavior to the solution. While the complex scaling method has been known for a long time, it was not been applied to scattering processes until Rescigno and coworkers implemented a cut-off strategy for the potential appearing in the driven term of the nonhomogeneous wave equation [19]. The exact solution of a two-body driven equation involving a Coulomb interaction and the strategy implemented within the framework of exterior complex scaling is object of our current investigations.

\section{ACKNOWLEDGMENTS}

One of the authors (G.G.) thanks the support by PGI (24/F049) of the Universidad Nacional del Sur, ANPCyT (PICT08/0934) (Argentina), and PIP 200901/552 CONICET (Argentina). The support provided by the Lorraine region for G.G.'s visit to the Laboratoire de Physique Moléculaire et des Collisions, Université Paul Verlaine-Metz, is also gratefully acknowledged.
[1] J. D. Jackson, Classical Electrodynamics (John Wiley \& Sons, New York, 1975).

[2] R. G. Newton, Scattering Theory of Waves and Particles (Dover, New York, 2002).

[3] C. J. Joachain, Quantum Collision Theory (North-Holland, Amsterdam, 1983).

[4] T. N. Rescigno, M. Baertschy, W. A. Isaacs, and C. W. McCurdy, Science 286, 2474 (1999).

[5] C. W. McCurdy, M. Baertschy, and T. N. Rescigno, J. Phys. B 37, R137 (2004)

[6] A. D. Alhaidari, E. J. Heller, H. A. Yamani, and M. S. Abdelmonem, eds., The J-Matrix Method, Development and Applications (Springer, Berlin, 2008).

[7] H. A. Yamani and W. P. Reinhardt, Phys. Rev. A 11, 1144 (1975).

[8] J. T. Broad, Phys. Rev. A 31, 1494 (1985).

[9] G. Gasaneo and L. U. Ancarani, Phys. Rev. A 80, 062717 (2009).

[10] B. H. Bransden and C. J. Joachain, Physics of Atoms and Molecules, 2nd ed. (Prentice Hall, Englewood Cliffs, NJ, 2003).
[11] L. D. Landau and E. M. Lifshitz, Quantum Mechanics: NonRelativistic Theory (Pergamon, Oxford, UK, 1965).

[12] A. Erdelyi, W. Magnus, F. Oberhettinger, and F. G. Tricomi, Higher Trascendental Functions (McGraw-Hill, New York, 1953), Vols. I, II, and III.

[13] M. Abramowitz and I. A. Stegun, Handbook of Mathematical Functions (Dover, New York, 1972).

[14] A. W. Babister, Transcendental Functions Satisfying Nonhomogeneous Linear Differential Equations (The Macmillan Company, New York, 1967).

[15] H. Buchholz, The Confluent Hypergeometric Function (Springer-Verlag, Berlin, 1969).

[16] L. U. Ancarani and G. Gasaneo, J. Math. Phys. 49, 063508 (2008).

[17] P. Appell and J. Kampé de Fériet, Fonctions Hypergéométriques et Hypersphériques; Polynomes d'Hermite (Gauthier-Villars, Paris, 1926).

[18] L. U. Ancarani and G. Gasaneo (submitted to J. Math. Phys).

[19] T. N. Rescigno, M. Baertschy, D. Byrum, and C. W. McCurdy, Phys. Rev. A 55, 4253 (1997). 